Available online on 15.04.2020 at http://jddtonline.info
Open Access to Pharmaceutical and Medical Research
unrestricted non-commercial use, provided the original work is properly cited

Open@ Access

Research Article

\title{
The Assessment of Mucoadhesivity of Natural Polymer Derived Form Plant Sources
}

\author{
Radheshyam Samanta $^{*}$, Asish Bhaumik ${ }^{2}$, Dr. Amit Kumar Nayak ${ }^{3}$ \\ ${ }^{1}$ Assistant Professor, Narayan Institute of Pharmacy, Gopal Narayan Singh University, Jamuhar-821305, Sasaram, Bihar, India \\ ${ }^{2}$ Associate Professor, Narayan Institute of Pharmacy, Gopal Narayan Singh University, Jamuhar-821305, Sasaram, Bihar, India \\ ${ }^{3}$ Associate Professor, Department of Pharmaceutics, Seemanta Institute of pharmaceutical Sciences, Jharpokharia, Mayurbhang-757086, \\ Odisha, India.
}

\section{ABSTRACT}

The main aim and objective of my present research work was to determine the various important mucoadhesive parameters such as mucoadhesive force, force of adhesion and bonding strength etc. Mucoadhesive properties of natural polymers were evaluated by formulating gels using Carbopol $940 \mathrm{P}$ as a gelling agent. Mucoadhesive parameters of the prepared Carbopol $940 \mathrm{P}$ gels containing natural polymers were determined by ex vivo followed by modified physical balance using excised cock intestinal mucosa. From the recent experimental data it was displayed that the mucoadhesive strength, force of adhesion and bonding strength of gel containing low methoxy pectin ( $1 \% \mathrm{w} / \mathrm{v}$ ) was found higher than other tested gels. The gel containing Moringa oleifera gum (1\% w/v) exhibited less mucoadhesion than other tested gels. The order of mucoadhesion of these plant-derived polymers was found as: low methoxy pectin $>$ jackfruit seed starch $>$ cashew gum/okra gum gum $>$ pumpkin pectin $>$ linseed mucilage $>$ sago starch $>$ gum Arabic $>$ xanthan gum $>$ fenugreek seed mucilage/black palm seed polysa ccharide $>$ Moringa oleifera gum.
\end{abstract}

Keywords: Mucoadhesion, Mucoadhesive strength, gelling agent, intestinal mucosa, and plant derived polymers etc.

Article Info: Received 24 Jan 2020; Review Completed 18 March 2020; Accepted 26 March 2020; Available online 15 April 2020

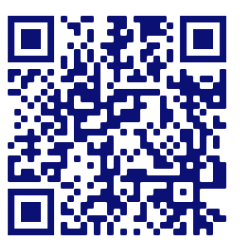

Cite this article as:

Samanta R, Bhaumik A, Nayak AK, The Assessment of Mucoadhesivity of Natural Polymer Derived Form Plant Sources, Journal of Drug Delivery and Therapeutics. 2020; 10(2-s):27-29 http://dx.doi.org/10.22270/jddt.v10i2-s.3952

*Address for Correspondence:

Radheshyam Samanta, Assistant Professor, Narayan Institute of Pharmacy, Gopal Narayan Singh University, Jamuhar821305, Sasaram, Bihar, India

\section{INTRODUCTION}

Mucoadhesion is defined as the attachment of synthetic or natural macromolecules to any mucus-lining present on biological epithelial surface [1]. It is mostly achieved by the use of mucoadhesive polymers [2-4]. Mucoadhesion is provided by the formation of non-covalent bonds such as hydrogen bonds and ionic interactions or physical entanglements between the mucus gel layer and polymers.

Classification of mucoadhesive polymers

The classification of various mucoadhesive polymers used in the formulation of various mucoadhesive systems is [1-5]:

(I) Synthetic polymers: (a) Cellulose derivatives: Methylcellulose, Ethylcellulose, Hydroxy-ethylcellulose, Hydroxyl propyl cellulose, Hydroxy propyl methylcellulose, Sodium carboxy methylcellulose. (b) Poly (acrylic acid) polymers: Carbomers, olycarbophil. (c) Poly (hydroxyethyl methylacrylate). (d) Poly (vinyl alcohol), etc.
(II) Natural polymers: (a) Sodium alginate. (b) Guar gum. (c) Xanthan gum. (d) Lectin. (e) Gelatin. (f) Pectin. (g) Chitosan etc.

Natural mucoadhesive polymers obtained from plant sources

The use of natural polymers in various biomedical and food continues to be an area of intensive research despite the advent of several new synthetic polymers [6]. Natural polymers primarily remain attractive for their capability of chemical modifications, biodegradability and biocompatibility [7-8]. Various conventional natural mucoadhesive polymers like guar gum, chitosan, xanthane gum, alginates, locust bean gum, alginate, etc, were already well studied and investigated in various drug delivery applications [6, 9-12].

In the literature, there are several reports on natural mucoadhesive agents, which were isolated from various plant materials and have found their use for the development 
of mucoadhesive systems. The examples of these newer natural mucoadhesive agents, which found their potential application in the development of mucoadhesive applications, are mucoadhesive agents isolated or extracted from: ispaghula husk [13-14], Assam Bora rice [15], Linseed [16], tamarind seed [17], Dellinia indica L. fruits [18], Trigonella foenum-graecum L. (fenugreek) seeds [19], Aegle marmelos fruits [20] etc.

\section{MATERIALS AND METHODS}

\section{Materials and chemicals}

Gum Arabic, xanthan gum, and low methoxy pectin were commercial available samples. Cashew gum, Moringa oleifera gum, okra gum, pumpkin pectin, fenugreek seed mucilage, linseed mucilage, black palm seed polysaccharide, jackfruit seed starch and sago starch were previously extracted in our laboratory. All other chemicals and reagents were commercially available and of analytical grade.

\section{Method}

\section{Formulation of isolated natural polymeric gels}

Mucoadhesive properties of natural polymers were evaluated by formulating gels using Carbopol $940 \mathrm{P}$ as a gelling agent. Natural polymers (namely, gum Arabic, xanthan gum, low methoxy pectin, cashew gum, Moringa oleifera gum, okra gum, pumpkin pectin, fenugreek seed mucilage, linseed mucilage, black palm seed polysaccharide, jackfruit seed starch and sago starch) were employed as mucoadhesive agents. Briefly, Carbopol $940 \mathrm{P}$ $(1.5 \% \mathrm{w} / \mathrm{v})$ was dispersed into aqueous solutions containing natural polymers $(1 \% \mathrm{w} / \mathrm{v})$. These dispersions were allowed to hydrate overnight followed by addition of triethanolamine $(0.1 \% \mathrm{w} / \mathrm{v})$ and glycerin $(0.5 \% \mathrm{w} / \mathrm{v})$ to form gels.

\section{Evaluation of mucoadhesive potential of gels}

Mucoadhesive parameters (mucoadhesive force, force of adhesion, and bonding strength) of the prepared Carbopol $940 \mathrm{P}$ gels containing natural polymers were assessed ex vivo by modified physical balance using excised cock intestinal mucosa. The modified physical balance apparatus was comprised of a two-arm balance, one side of which was contained two glass plates (lower plate) was attached permanently to the base of the stage, and the other (upper plate) was glued to the base of one arm of the balance. The membrane used for mucoadhesive testing was fresh cock intestine, which was glued to the lower plate and another was glued to the upper plate by using cyanoacrylate adhesive. Accurately weighed, 1 gram of gels was placed on the goat intestinal mucosa glued to the upper side of the lower plate. Then, the upper plate was placed over the lower plate. A contact pressure by the figure tip was applied for 5 minutes (preload time). After removal of the preload force, a gradually increasing weight was applied on the second arm of the balance by controlled addition of water from the burette till the plates detached from each other. The weight required for the detachment of the glass plates was recorded and the mucoadhesive strength of the prepared gels was calculated [20]. From mucoadhesive strength values (in gram), force of adhesion (Newton) and bonding strength (Newton/meter ${ }^{2}$ ) were easily calculated. Mucoadhesive parameters will be evaluated are as follows: Mucoadhesive strength $=$ the mass (in gram) required to detach the polymer sample from the mucosal surface.

Force of adhesion $(N)=\frac{(\text { Mucoadhesive strength } \times 9.81)}{1000}$

Bonding strength $\left(\frac{N}{M^{2}}\right)=\frac{(\text { Force of adhesion })}{(\text { Mucosal surface area })}$

\section{RESULTS AND DISCUSSION}

Table-1: Mucoadhesive parameters of various gels containing isolated natural polymers $(1 \% \mathrm{w} / \mathrm{v})$

\begin{tabular}{|c|c|c|c|}
\hline Gels & $\begin{array}{l}\text { Mucoadhesive } \\
\text { strength (gr) }\end{array}$ & $\begin{array}{c}\text { Force of } \\
\text { adhesion }(\mathrm{N})\end{array}$ & $\begin{array}{c}\text { Bonding strength } \\
\left(\mathrm{N} / \mathrm{M}^{2}\right)\end{array}$ \\
\hline Gel containing gum Arabic $(1 \% \mathrm{w} / \mathrm{v})$ & 15.20 & $1.49 \times 10^{-4}$ & 1.90 \\
\hline Gel containing xanthan gum $(1 \% \mathrm{w} / \mathrm{v})$ & 15.10 & $1.48 \times 10^{-4}$ & 1.88 \\
\hline Gel containing low methoxy pectin (1\% w/v) & 21.00 & $2.05 \times 10^{-4}$ & 2.60 \\
\hline Gel containing isolated cashew gum (1\% w/v) & 18.60 & $1.82 \times 10^{-4}$ & 2.32 \\
\hline Gel containing Moringa oleifera gum (1 \% w/v) & 14.00 & $1.37 \times 10^{-4}$ & 1.74 \\
\hline Gel containing isolated okra gum (1\% w/v) & 18.60 & $1.82 \times 10^{-4}$ & 2.32 \\
\hline Gel containing isolated pumpkin pectin $(1 \% \mathrm{w} / \mathrm{v})$ & 18.43 & $1.82 \times 10^{-4}$ & 2.32 \\
\hline Gel containing isolated fenugreek seed mucilage $(1 \% \mathrm{w} / \mathrm{v})$ & 14.50 & $1.42 \times 10^{-4}$ & 1.80 \\
\hline Gel containing linseed mucilage $(1 \% \mathrm{w} / \mathrm{v})$ & 16.83 & $1.64 \times 10^{-4}$ & 2.08 \\
\hline Gel containing isolated black palm seed polysaccharide $(1 \% \mathrm{w} / \mathrm{v})$ & 14.50 & $1.42 \times 10^{-4}$ & 1.80 \\
\hline Gel containing isolated jackfruit seed starch $(1 \% \mathrm{w} / \mathrm{v})$ & 19.60 & $1.92 \times 10^{-4}$ & 2.44 \\
\hline Gel containing isolated sago starch $(1 \% \mathrm{w} / \mathrm{v})$ & 15.43 & $1.51 \times 10^{-4}$ & 1.92 \\
\hline
\end{tabular}


From the recent experimental data (Table-1), it was found that the mucoadhesive strength, force of adhesion and bonding strength of gel containing low methoxy pectin $(1 \%$ $\mathrm{w} / \mathrm{v}$ ) was found higher than other tested gels. The gel containing Moringa oleifera gum ( $1 \% \mathrm{w} / \mathrm{v})$ exhibited less mucoadhesion than other tested gels. The order of mucoadhesion of these plant-derived polymers was found as: low methoxy pectin $>$ jackfruit seed starch $>$ cashew gum/okra gum $>$ pumpkin pectin $>$ linseed mucilage $>$ sago starch $>$ gum Arabic $>$ xanthan gum $>$ fenugreek seed mucilage/black palm seed polysaccharide > Moringa oleifera gum. Actually, native natural polymers are able to hydrate rapidly and bind with the mucous layer through the hydrogen bonding, which could be the mechanism of the mucoadhesion of these gels containing natural polymers as mucoadhesive agents.

Thus, mucoadhesivity of these natural polymers obtained from plant sources prompted us to explore for mucoadhesivity in biomedical and pharmaceutical applications.

\section{CONCLUSION}

From the current investigation here we reported that some selected natural polymers isolated from plant sources namely, gum Arabic, xanthan gum, low methoxy pectin, cashew gum, Moringa oleifera gum, okra gum, pumpkin pectin, fenugreek seed mucilage, linseed mucilage, black palm seed polysaccharide, jackfruit seed starch and sago starch were evaluated for their mucoadhesive potential. To explore their mucoadhesive applications, Carbopol 940 P (1.5\% $\mathrm{w} / \mathrm{v}$ )-based mucoadhesive gels containing these natural polymers $(1 \% \mathrm{w} / \mathrm{v})$ as mucoadhesive agents were formulated and evaluated for their various important mucoadhesive parameters like mucoadhesive strength, force of adhesion and bonding strength. From this investigation, it was observed that the mucoadhesive strength, force of adhesion and bonding strength of gel containing commercially available low methoxy pectin $(1 \% \mathrm{w} / \mathrm{v})$ were found higher than these of the other gels tested. The promising mucoadhesive properties of these natural mucoadhesive polymers isolated from commonly available sources warrant their further exploration for the use in various mucoadhesive applications in the biomedical field.

\section{REFERENCES}

[1] Chowdary KPR, Srinivas L. Mucoadhesive drug delivery systems: a status of current review. Indian Drugs 2000; 37: 400-406.

[2] Patil SB, Murthy RSR, Mahajan HS, Wagh RD, Gattani SG. Mucoadhesive polymers: Means of improving drug delivery. Pharma Times 2006; 38: 25-28.

[3] Pal D, Nayak AK. Development, optimization, and anti-diabetic activity of gliclazide-loaded alginate-methyl cellulose mucoadhesive microcapsules. AAPS PharmSciTech 2011; 12: 1431-1441.

[4] Pal D, Nayak AK. Novel tamarind seed polysaccharide-alginate mucoadhesive microspheres for oral gliclazide delivery: In vitro-in vivo evaluation. Drug Deliv 2012; 19: 123-131.

[5] Chowdary KPR, Srinivas Rao S. Mucoadhesive microspheres and microcapsules: Current status. Indian J Pharm Sci 2005; 67: 141-150.

[6] Kibbe AH. Handbook of pharmaceutical excipients. Pharmaceutical Press: Washington, D.C. USA, 2000: pp. 102-109.

[7] Pal D, Nayak AK, Kalia S. Studies on Basella alba L. leaves mucilage: Evaluation of suspending properties. Int J Drug Discov Tech 2010; 1(1): 15-20.

[8] Nayak AK, Pal D, Pany DR, Mohanty B. Evaluation of Spinacia oleracea L. leaves mucilage as innovative suspending agent. J Appl Pharm Tech Res 2010; 1(3): 338-341.

[9] Jani GK, Shah DP, Prajapati VD, Jain V. Gums and mucilages: versatile excipients for pharmaceutical formulations. Asian J Pharm Sci. 2009; 4(5): 309-323.

[10] Avachat AM, Dash RR, Shrotriya SN. Recent investigations of plant based natural gums, mucilages and resins in novel drug delivery systems. Indian J Pharm Educ Res. 2011; 45(1): 86-99.

[11] Barbosa MA, Granja PL, Barrias CC, Amaral IF. Polysaccharides as scaffolds for bone regeneration. ITBM-RBM 2005; 26: 212217.

[12] Patil SB, Murthy RSR, Mahajan HS, Wagh RD, Gattani SG. Mucoadhesive polymers: Means of improving drug delivery. Pharma Times 2006; 38(4): 25-28.

[13] Nayak AK, Hasnain MS, Beg S, Alam MI. Mucoadhesive beads of gliclazide: Design, development, and evaluation. Sci Asia 2010; 36: 319-325.

[14] Sharma VK, Bhattacharya A. Release of metformin hydrochloride from ispaghula-sodium alginate beads adhered on cock intestinal mucosa. Indian J Pharm Educ Res 2008; 42 (4): 365-372.

[15] Sachan NK, Bhattacharya A. Evaluation of Assam Bora rice starch as a possible natural mucoadhesive polymer in formulation of microparticulate controlled drug delivery systems. J Assam Sci Soc 2006; 47: 34-41.

[16] Basu S, Chakraborty S, Bandyopadhyay AK. Development and evaluation of a mucoadhesive nasal gel of midazolam prepared with Linum usitatissimum L. seed mucilage. Sci Pharm 2009; 77: 899-910.

[17] Gheraldi E, Tavanti A, Celandroni F, Lupetti A, Blandizzi C, Boldrini E, Campa M, Senesi S. Effect of novel mucoadhesive polysaccharide obtained from tamarind seeds on the intraocular penetration of gentamicin and ofloxacin in rabbits. J Antimicrob Chemother 2000; 46: 831-834.

[18] Ketousetuo K, Bandyopadhyay AK. Development of oxytocin nasal gel using natural mucoadhesive agent obtained from the fruits of Dellinia indica. L. Sci Asia 2007; 33: 57-60.

[19] Dutta R, Bandyopadhyay AK. Development of a new nasal drug delivery system of diazepam with natural mucoadhesive agent from Trigonella foenum-graecum L. J Sci Ind Res 2005; 64: 973977.

[20] Chanda R, Nath LK, Mahapatra SK. Formulation development of oral mucoadhesive coated terbutaline sulphate tablets using some natural materials extracted from edible fruits available in India. Iranian J Pharm Res 2009; 5(1): 3-12. 